\title{
Plasma rotation induced by the Dynamic Ergodic Divertor
}

\author{
K.H. Finken ${ }^{\mathrm{a}}$, S.S. Abdullaev ${ }^{\mathrm{a}}$, T. Eich ${ }^{\mathrm{a}}$, D.W. Faulconer ${ }^{\mathrm{b}}$, M. Kobayashi ${ }^{\mathrm{a}, \mathrm{c}}$, \\ R. Koch ${ }^{\mathrm{b}}$, G. Mank ${ }^{\mathrm{a}}$, A. Rogister ${ }^{\mathrm{a}}$ \\ ${ }^{a}$ Institut für Plasmaphysik, Forschungszentrum Jülich GmbH, Euratom Association \\ (Partner in the Trilateral Euregio Cluster), \\ Jülich, Germany \\ ${ }^{\mathrm{b}}$ Ecole Royale Militaire - Koninklijke Militaire School, Association Euratom-Belgian State \\ (Partner in the Trilateral Euregio Cluster), \\ Brussels, Belgium \\ ${ }^{c}$ Department of Energy Engineering and Science, Nagoya University, \\ Nagoya, Japan
}

\begin{abstract}
After introduction of the experimental options available with the Dynamic Ergodic Divertor (DED) and a discussion of the static aspects of the ergodic and laminar zones, the dynamic aspects of the rotating DED field are emphasized. The rotating perturbation field induces a shielding current which is modelled under different assumptions. Interaction of the shielding current with that of the DED coils results in a torque exerted by the coils on the plasma. The location of the maximum of this torque with respect to the frequency depends critically on the width of the shielding current, and for the TEXTOR-DED conditions it is in the frequency band of $1-30 \mathrm{kHz}$. The DED will have the option of operation with full power in this band so that the basic investigations on the field line penetration can be attempted. The force transferred to the plasma has two components, a weaker toroidal one and a dominant poloidal one. The toroidal force component has about the same value as the one from NBI; from the experience with NBI induced plasma rotation, a substantial plasma acceleration in the toroidal direction is expected. For neoclassical reasons it is not yet clear whether the dominant poloidal force component will result in a poloidal plasma rotation or a radial force. If the poloidal rotation is inhibited, a static radial electric field is estimated on the basis of a revisited neoclassical theory to be of the order of several kilovolts per metre.
\end{abstract}

\section{Introduction}

Progress in plasma confinement over recent decades has been based to a large extent on the careful design of magnetic flux surfaces. The sufficiently low error margin from the different devices makes it now possible to extrapolate confinement data to a reactor scenario such that it can be expected that the plasma of the proposed ITER experiment will most likely ignite. The basic feature of the magnetic flux surfaces is their onion-like structure. Magnetic field lines stay always on 'their' magnetic surface and for a non-streaming plasma these surfaces also form isobars. Nevertheless, the existence of flux surfaces is generally not guaranteed; other, namely 'ergodic', solutions for the magnetic field line structure are possible as well. Ergodicity in this particular case means that any magnetic field line comes infinitely close to any point in the given ergodic volume. This property is incompatible with flux surfaces where a field line is restricted to one surface.

The formation of an ergodic layer can be an interesting means of destroying the good confinement properties of closed flux surfaces at special locations. Such a location of interest is the boundary of the plasma. All power, with the exception of the radiated fraction, introduced into the plasma either by heating or by particles, which leaves the plasma conductively or convectively passes through a relatively thin boundary layer. Even in a fusion reactor, this scrape-off layer will be only one to several centimetres thick. The highly concentrated heat flux traversing this layer follows the magnetic field lines and hits the divertor target plate or the limiter surface. It has been shown that the heat flux density is extremely high for any material to handle. To mitigate the problem, the concept of a radiative mantle has been introduced and basic investigations have been 


\section{K.H. Finken et al.}

performed on the TEXTOR tokamak, for example. A similar goal, namely to distribute the heat flux over a large wall area, is shared by the new TEXTOR proposal, the Dynamic Ergodic Divertor (DED) [1] which is presently under construction. The DED will create an ergodic boundary layer of the plasma and thus it is expected to allow an improved screening of impurities, an optimized use of noble impurities under high performance RI mode operation and an enhanced particle removal by the pump limiter ALTII. The expression 'dynamic' refers to a rotating perturbation magnetic field imposed by the DED coils. For the rotation, different frequencies are foreseen, such as a few hertz for distribution of the heating pattern of the divertor strike zones over the large area of the divertor target plate, or frequencies of up to $10 \mathrm{kHz}$ which can lead to an unlocking of modes, or impose a differential rotation in the plasma edge and improve confinement.

In this context, the high frequency operation of the DED at frequencies of between 1 and $10 \mathrm{kHz}$ is of particular interest. The 4 phase AC current in the DED coils generates a rotating magnetic field pattern which imposes a torque at the plasma edge. The force underlying the torque is directed mainly in the poloidal direction while a relatively small component $(10 \%)$ is oriented toroidally; this force generates a radial electric field and a rotation of the plasma. In the following, after a description of the DED system, the static ergodization pattern is shown. This is the starting point for considerations on the rotating DED field. The rotating field induces shielding currents in the highly complicated ergodic structure, the physics of magnetic field line reconnection in this region being a very new field for investigation where little is known. This is followed by results obtained relating to the dynamic effect of the shielding currents, namely the forces imposed by the external DED field on the plasma. Different models of the magnetic field line reconnection physics in the ergodic layer have been applied in order to calculate these forces. Finally, estimates of the expected electric field strength and of the induced toroidal rotation are topics of this article.

\section{Experimental set-up}

The DED is a new conceptual approach which will be installed in TEXTOR-94 in the year 2001. The main component of the DED is a set of magnetic perturbation coils whose purpose is to ergodize the magnetic field structure in the plasma edge region;

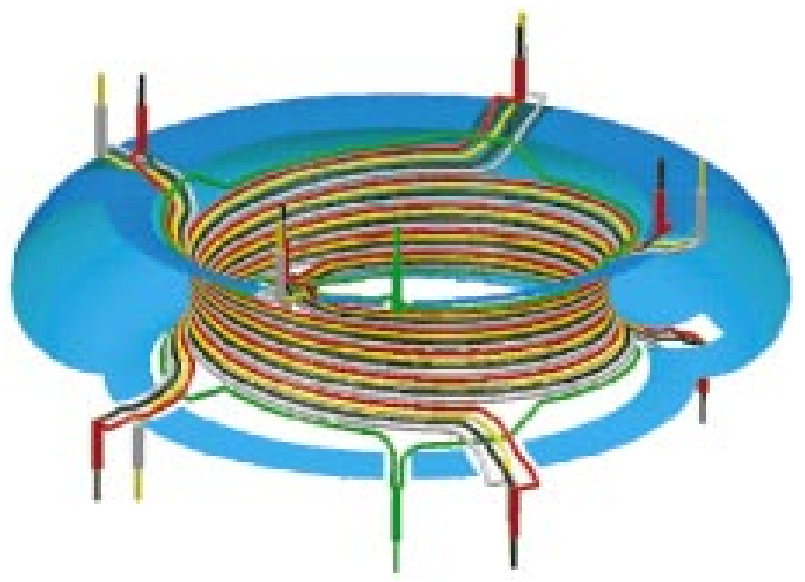

Figure 1. Experimental set-up of the DED coils. The perturbation coils are located inside the vacuum vessel at the high field side. Each coil goes helically once around the torus and is oriented parallel to the magnetic field lines of the $q=3$ surface.

these coils are located inside the vacuum vessel at the high field side of the torus, as shown in Fig. 1. The set consists of 16 individual coils (four quadruples) plus two compensation coils. The individual perturbation coils, each winding once around the torus, follow the direction of the equilibrium magnetic field of the plasma edge (i.e. helically); the radial location of enhanced interaction where the helical pitch of the coils exactly matches that of the equilibrium field can be fine tuned, for example by varying the plasma current. By this means, a resonant effect of the external perturbation field is obtained on the edge plasma at a preselected radius whereby a perturbation current of only $15 \mathrm{kA}$ is sufficient to create a stochastic structure.

The five main perturbation modes (i.e. poloidal and toroidal Fourier mode combinations) are centred at $m / n=12 / 4$; this has been selected because it creates only small local perturbations (magnetic islands) and avoids undesired disturbances in the plasma core. By connecting certain perturbation coils in series, the use of lower $m$ and $n$ is possible, which is of interest for exciting and systematically analysing modes located deeper inside the torus. In addition, the power supplies are laid out to allow a superposition of the base $12 / 4$ mode with either the $6 / 2$ mode or the $3 / 1$ mode. This option allows to some degree a decoupling of the perturbation strength at the plasma edge from its penetration depth into the plasma. 
The DED has the unique feature that the perturbation field is not static as in most other devices but has the option of rotation. To our knowledge only the small research tokamak CSTN [2] at Nagoya University has similar features and, at low perturbation current levels, also the TEXT [3] tokamak. The DED can be operated $\mathrm{DC}$, at around $50 \mathrm{~Hz}$ or at seven frequencies in the band from 1 to $10 \mathrm{kHz}$. At low perturbation current $(1.5 \mathrm{kA})$, the perturbation field can be applied across the whole frequency band of interest for feedback stabilization experiments.

\section{Modelling of the static ergodic layer}

The perturbation coils impose structures with different properties in the plasma boundary. The interior closed flux surfaces, $q(r) \leq 2$, remain nearly undisturbed and form for at least well structured KAM [4] surfaces. Further towards the boundary, islands develop and start to grow; finally, different islands overlap and form an ergodic sea [5-7], such that the magnetic surfaces are destroyed. The very outermost magnetic field lines intersect the divertor target plate. These field lines form the 'laminar zone' [8]; here the transport changes character from diffusive to convective with plasma streaming to the plasma facing components as in the conventional scrape-off layer.

Field line tracing and field line mapping $[9,10]$ are basic techniques for investigating the ergodic structure. These techniques provide a picture of the areas with intact magnetic surfaces, of island structures and of ergodized regions. Beyond this, the radial displacement of the field lines has been evaluated in a statistical sense and the field line diffusion coefficient as a function of the radius has been derived. The resonant radius can be adjusted via the plasma current; in this way the ergodic zone can be shifted radially in the range $0.9 \leq r / a \leq 1$ keeping the Chirikov parameter above one.

Individual adjustment of the coil currents allows the superposition [11] of different external base modes. To minimize the interaction with the core plasma, high $m / n$ (e.g. $m / n=12 / 4$ ) numbers are preferred; for the given coil arrangement, Fourier mode numbers $m \leq 8$ can be neglected. This choice creates many small islands instead of a few islands with large poloidal extent. The rapid decay of the perturbation amplitude with distance from the coils is a problem, however. This decay can be compensated by an admixture of the $m / n=6 / 2$ mode with the base $12 / 4$ mode. This mode mixing will provide the highest ergodization levels in TEXTOR-94, with a Chirikov parameter above four at the plasma edge and remaining larger than one over a $6 \mathrm{~cm}$ wide layer. The maximum tolerable admixture of the $6 / 2$ mode is judged to be about $30 \%$ because from this value on, the $q=2$ surface will merge into the ergodic sea.

Figures 2(a)-(c) show Poincaré plots for different plasma currents $(460,530$ and $600 \mathrm{kA})$ at full perturbation current. With increasing plasma current, the resonant surfaces $(2.25 \leq q \leq 3.75)$ move towards the outside, closer to the DED coils. In addition, the amplitude of the perturbation field increases with decreasing separation of the resonances from the perturbation coils [11]; the amplitude varies roughly with a power of $B_{r} \propto r^{m_{\text {eff }}}, m_{\text {eff }} \approx 20$. Therefore the highest ergodization levels, with Chirikov parameters of up to 6 , are expected for plasma currents of around $600 \mathrm{kA}$. The results of the field line mapping seem at first glance to be in conflict with this expectation: the radially widest ergodic zone is found at the relatively low plasma current of $460 \mathrm{kA}$, while at $600 \mathrm{kA}$ the properly ergodic zone is only a small band at a radius at about $r=44 \mathrm{~cm}$. This 'inconsistency' will be removed by considerations on the laminar zone given below. At the intermediate plasma current (Fig. 2(b)) the ergodic zone also shows a medium extent in width.

The outer zones of Figs 2(b) and (c) are nearly devoid of Poincaré intersection points and appear therefore as white. The absence of the intersection points is a consequence of stopping the field line tracing after an intersection with a wall element (predominantly with the divertor target plate in front of the coils). The reason for this stopping is that particles following field lines would be neutralized and could leave their field line. The 'white' area obviously contains field lines with a short connection length. This area of short connection length is called the laminar zone $[12,13]$. Because of its short connection length, the laminar zone is more related to the scrape-off layer than to the properly ergodic zone with very long field line connection lengths. The attempt to increase the ergodic zone by increasing the resonant perturbation at the edge evidently leads more to an increase of the laminar layer than to a widening of the ergodic zone.

A quantity showing the relevant transport character of the ergodic zone is the local field line diffusion coefficient $D_{F L}$; it is defined by the mean radial 

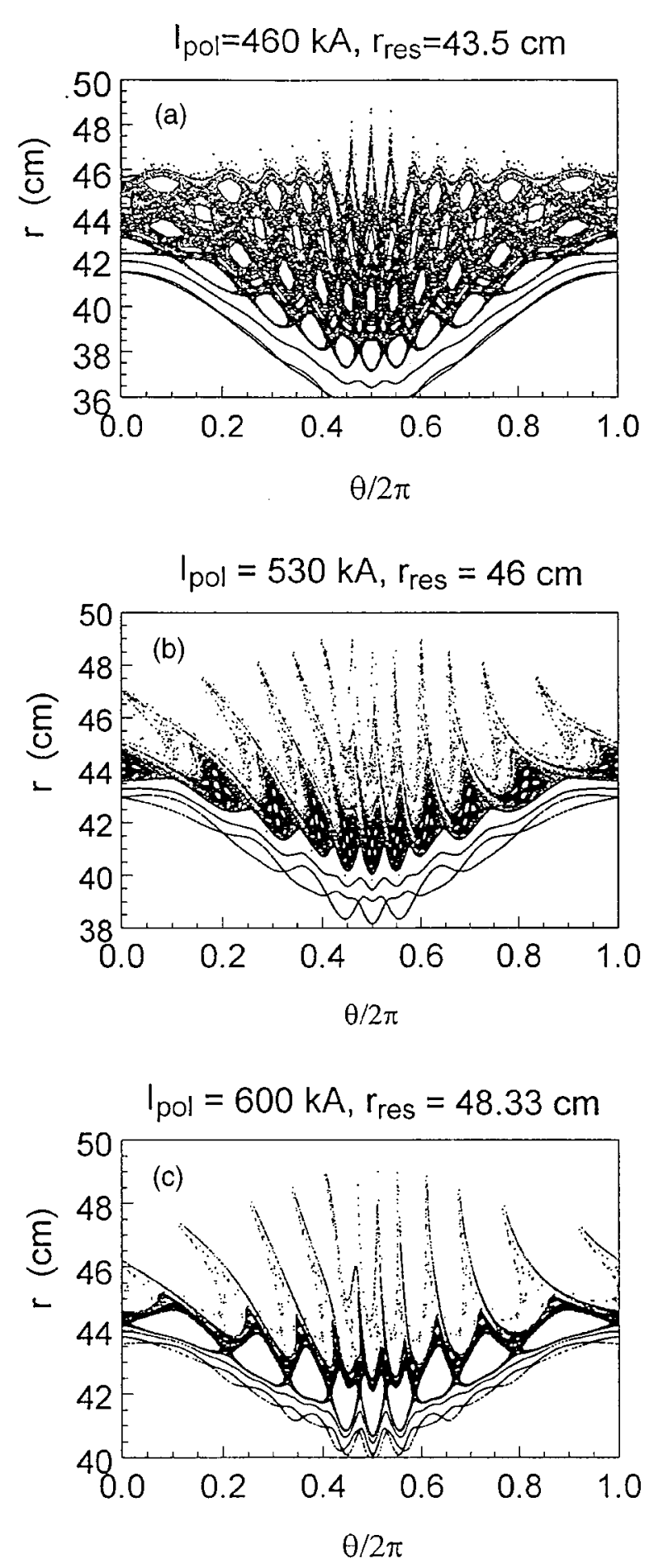

Figure 2. Variation of the ergodization pattern with increasing plasma current (at full perturbation current): (a) $I_{p}=460 \mathrm{kA}$, (b) $I_{p}=530 \mathrm{kA}$ and (c) $I_{p}=600 \mathrm{kA}$. Owing to the shift of the resonant $q$ surfaces towards the DED coils, the edge Chirikov parameter increases, leading to an opening of the laminar zone (field lines with short connection length). displacement of the field lines at a given radius for the initial few steps of the field line mapping. Figure 3 shows $D_{F L}$ as a function of the radius for the three plasma currents of Fig. 2. Coming from the plasma interior, the $D_{F L}$ curves start to increase. The values for $I_{p}=460 \mathrm{kA}$ are rather moderate over the whole radius. The other curves, $I_{p}=530 \mathrm{kA}$ and $I_{p}=600 \mathrm{kA}$, show a pronounced maximum at $44 \leq r \leq 45 \mathrm{~cm}$ and a four times higher amplitude. The decrease of the curves proceeding left from the maximum is expected from chaos theory in agreement with the value of the Chirikov parameter. The decay to the right of the maximum reflects the onset of a different physics mechanism, namely the presence of the laminar zone: the transport here no longer has a diffusive character but must be described by convective/conductive processes along the magnetic field lines in the laminar zone. As already stated, the enhanced perturbation at the plasma edge no longer enhances the diffusion coefficient but leads to widening of the laminar zone. The particle diffusive transport in the ergodic zone $\left(D_{\text {ions }}=D_{F L} c_{s}, c_{s}\right.$ equals the sound velocity) reaches at most a few $\mathrm{m}^{2} / \mathrm{s}$, the same order of magnitude as the typical anomalous transport at the plasma edge. The steep part of the slope of the curve for the highest plasma current is slightly shifted to the outside with respect to the slope of the curve for $I_{p}=530 \mathrm{kA}$. This shift results from the outward shift of the resonances with plasma current increase.

\section{Dynamic aspects}

The high frequency aspect of the DED field has been analysed in cylindrical approximation [14]. It has been shown that the 'low frequency' (relative to $\Omega_{i}$ ) electromagnetic wave of the DED effectively propagates in the area between coils and resonance layer as the compressional Alfvén wave (fast wave) [15]. At the resonance layer of the plasma different approximations have been made [14]. The interaction layer is described by an annulus of finite resistivity which is a function of the local electron temperature (skin effect). A theoretical uncertainty is the question of the radial width of this layer $(2 \varepsilon)$. One reasonable choice is the width of the ergodic zone derived from the Poincaré plots, which gives a typical value of $2 \varepsilon=2.5 \mathrm{~cm}$ (a); under a second assumption (b) the characteristic shielding width has been taken as the width of the statically calculated islands undiminished by overlapping (typically $0.5 \mathrm{~cm}$ ). This width is compatible with experiments made on TEXT [3] 


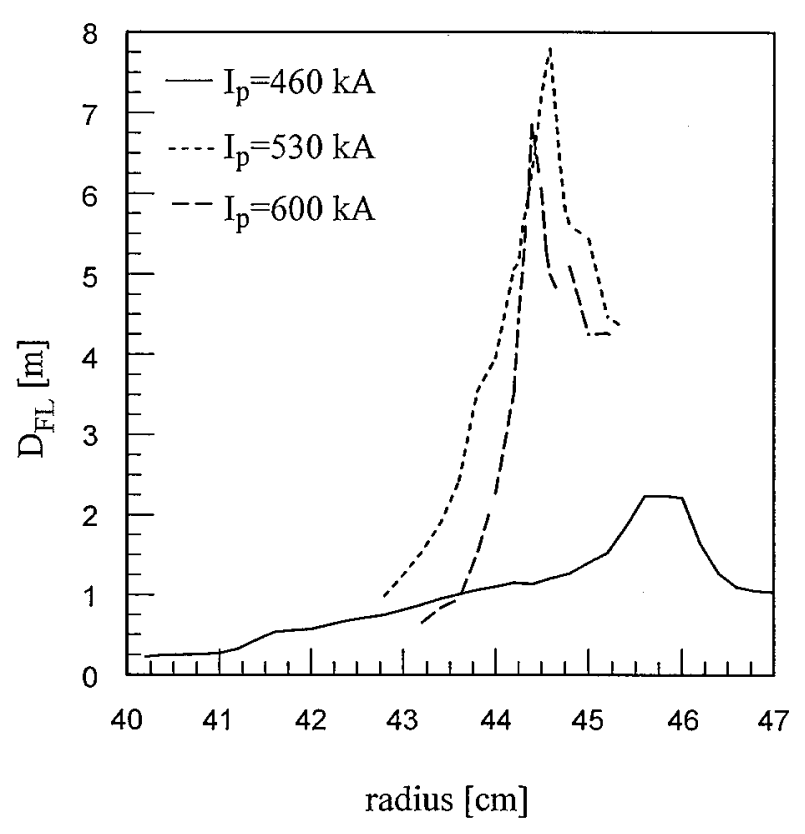

Figure 3. Local field line diffusion coefficient $D_{F L}$ as a function of the radius for cases (a)-(c) of Fig. 2.

for a situation of not fully ergodized edge field. Under a third assumption (c) a linear MHD theory has been applied which results in a shielding thickness of less than a millimetre.

It has also been shown that the interaction of the external rotating field with the current driven in the shielding layer results in the transfer of angular momentum between DED coil and plasma [14]. The maximum poloidal torque applied to the plasma amounts to about $100 \mathrm{Nm}$; this maximum occurs at a frequency which seems to depend mainly on the width of the current layer. In detail it depends on the assumed plasma temperature, on the applied frequency and on the assumed island or ergodization width. Examples (a)-(c) of the torque calculated for different conditions of the shielding current width are shown in Fig. 11 of Ref. [14]. Even if a poloidal rotation of the plasma were limited by neoclassical effects, the toroidal projection of the applied force would still be somewhat larger than that imposed by tangential NBI in TEXTOR, however, using $r$ instead of $R$ to compare the relative forces and (nonangular) momenta. This beam injection generates a toroidal rotation of more than $10^{7} \mathrm{~cm} / \mathrm{s}$. A combined operation of the high frequency DED with NBI will allow imposing interesting differential rotations between the plasma edge (action of the DED) and the core (by NBI).
Recently model (c) has been improved by taking into account the interaction of the background plasma equilibrium current with the wavefield. As in the previous attempt, the plasma is described by the single fluid MHD equations. The essential term for the plasma interaction stems from the linearized radial component of the oscillating plasma velocity:

$-\rho \frac{\partial v_{r}}{\partial t}=i \omega \rho v_{r}=\frac{\partial p}{\partial r}+j_{z}^{(1)} B_{\Theta}^{(0)}+j_{z}^{(0)} B_{\Theta}^{(1)}$.

The new term taken into account is the rightmost one containing the equilibrium current density $j_{z}^{(0)}$. We neglect the pressure term as in our previous treatment - the neglect of the pressure term in the ergodic zone is consistent with experimental data from Tore Supra - and obtain finally a differential equation of the form:

$$
\begin{gathered}
\frac{1}{r}\left(\frac{\partial}{\partial r} r \frac{\partial A_{z}}{\partial r}\right)-\frac{m^{2}}{r^{2}} A_{z}+\frac{1}{\delta^{2}+\left(v_{A, h e l} / \omega\right)^{2}} A_{z} \\
+\frac{1}{\left(\omega \delta / v_{A, h e l}\right)^{2}+1} \frac{\mu_{0} j_{z}^{0}}{B_{\Theta}(1-n q / m)} \frac{\partial A_{z}}{\partial r}=0 .
\end{gathered}
$$

Here $A_{z}$ is the only relevant component of the vector potential,

$\delta^{2}=\frac{1}{i \mu_{0} \sigma \rho}$

is the complex skin parameter,

$v_{A, h e l}^{2}=\frac{B_{\Theta}^{* 2}}{\mu_{0} \rho}$

is the Alfvén velocity based on the equilibrium field component normal to the direction taken by the field at resonance on the rational flux surface $q=3: B_{\Theta}^{*}=$ $B_{\Theta}(1-n q / m)$, and $\omega$ is the perturbation frequency.

Two terms determine the shape of the solution of the partial differential equation:

- The third term reflects the current of the driven wave, its coefficient having a resonance type character. It is only large if $v_{A, h e l} / \omega \leq|\delta|$; in the case of the TEXTOR DED conditions, this relation is only fulfilled over a radial width of $0.2 \mathrm{~mm}$.

- The new term entering the equation is the last one; it stems from the rightmost term of Eq. (1) and represents the interaction of the perturbation field with the equilibrium plasma current. The importance of this term depends critically on the equilibrium plasma current profile. 


\section{K.H. Finken et al.}

Therefore a parameter scan of the current distribution has been performed which is assumed to be of the type [16]:

$j_{e q}=j_{z}^{(0)}=\frac{(\nu+1) I_{p}}{\pi a^{2}}\left[1-\left(\frac{r}{a}\right)^{2}\right]^{\nu}$.

The parameter $\nu$ regulates the peakedness of the plasma current distribution.

$\nu=\frac{q_{a}}{q_{0}}-1$

i.e. $\nu \approx 4$ for $q_{a}=4$ and $q_{0}=0.8$ as typical operating conditions.

Since

$\mu_{0} j_{z}=(\nabla \times \nabla \times A)_{z}=\frac{1}{r}\left(\frac{\partial}{\partial r} r \frac{\partial A_{z}}{\partial r}\right)-\frac{m^{2}}{r^{2}} A_{z}$

one can split the shielding current into two terms $j=f 2+f 3$, where $f 2$ and $f 3$ are the expressions just discussed. Figure 4 shows the applied torque for three different choices of $\nu$, namely $\nu=1,2$ and 5 . Only for the relatively broad current distribution of $\nu=1$ does one see an effect of the equilibrium current distribution on the shift and broadening of the curve.

Figure 5 shows the force contributions of the 'resonant' term of Eq. (2) $\left(f 2 \times B_{r}\right.$, solid line $)$ and of the interaction term involving the equilibrium current $\left(f 3 \times B_{r}\right.$, dotted line) for $\nu=1$ and $\nu=2$. One sees clearly that the relative contribution from the interaction term resulting from $j_{z, \text { equi }} B_{\Theta}^{(1)}$ as compared with the $f 3 \times B_{r}$ part decreases strongly for a peaked current distribution. The detailed calculations show that the contribution of the interaction term is rather broad for $\nu=1$. In agreement with the results obtained from the variation of the width of the interaction zone of our previous model [14], the increase of the shielding current layer leads to a decrease of the maximum of the torque with respect to the frequency. At this maximum, the phase shift of the shielding current always assumes a value in the range $50-70^{\circ}$. Even though the relative contributions of the $f 2$ and $f 3$ terms change with $\nu$, the total force remains the same to within a factor of 2 .

Judging from Figs 4 and 5 one would conclude that the effect of equilibrium current on the shielding and on the torque can be neglected for typical DED operation. However, one future scenario involves the excitation of more internal modes in order to study mode physics in a reproducible way. In this case, the interaction with the background current will indeed be important.

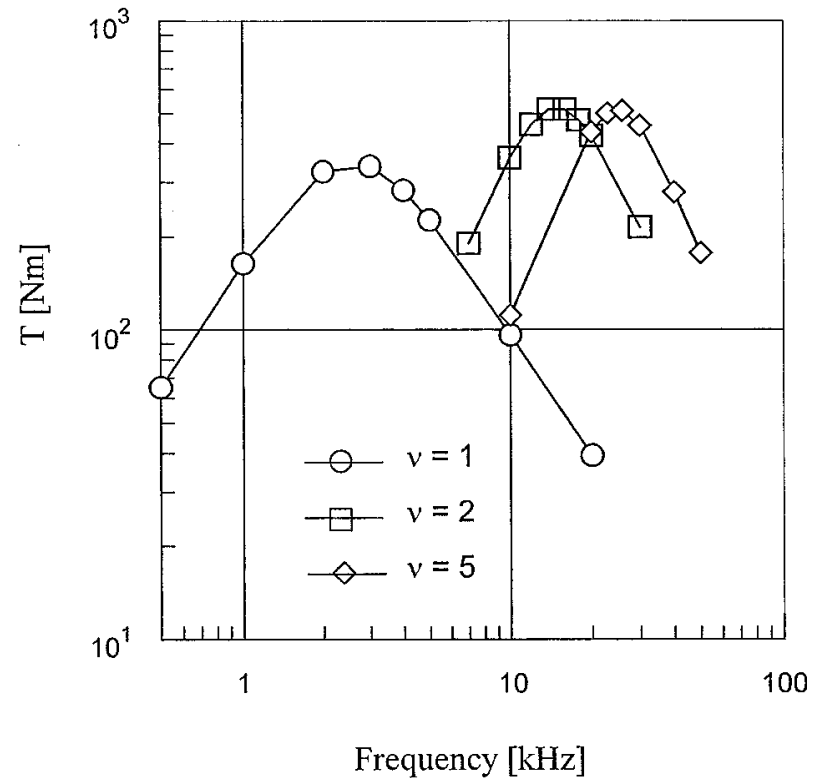

Figure 4. Torque exerted by the perturbation field on the plasma. The different curves represent the choices of the plasma current peaking exponent $\nu=1,2$ and 5 . Only for $\nu=1$ is the current distribution wide enough to give an important contribution from its interaction with the DED field.

\section{Estimate of the toroidal plasma rotation}

The velocity pattern and the electric field strength are closely coupled. In many cases (sheared) velocity distribution is of prime interest because it is presumed to be the main cause for the destruction of the convective cells [17] in the plasma that are identified as one of the major channels of energy loss in a tokamak. Barriers inside the plasma or at the plasma edge are explained in this way.

The driving force for plasma rotation is the torque applied by the rotating external DED field on the plasma. We consider the effect of the underlying force component in the toroidal direction separately from that in the poloidal direction because different models have to be considered. According to the results from conventional neoclassical theory, a free toroidal motion of the plasma is allowed and the accelerating force is balanced by a viscous force such that we can write:

$\nu^{*} v=\frac{F_{t}}{M}$.

Here $\nu^{*}$ is the rate with which the particles near the boundary exchange momentum with the wall. 

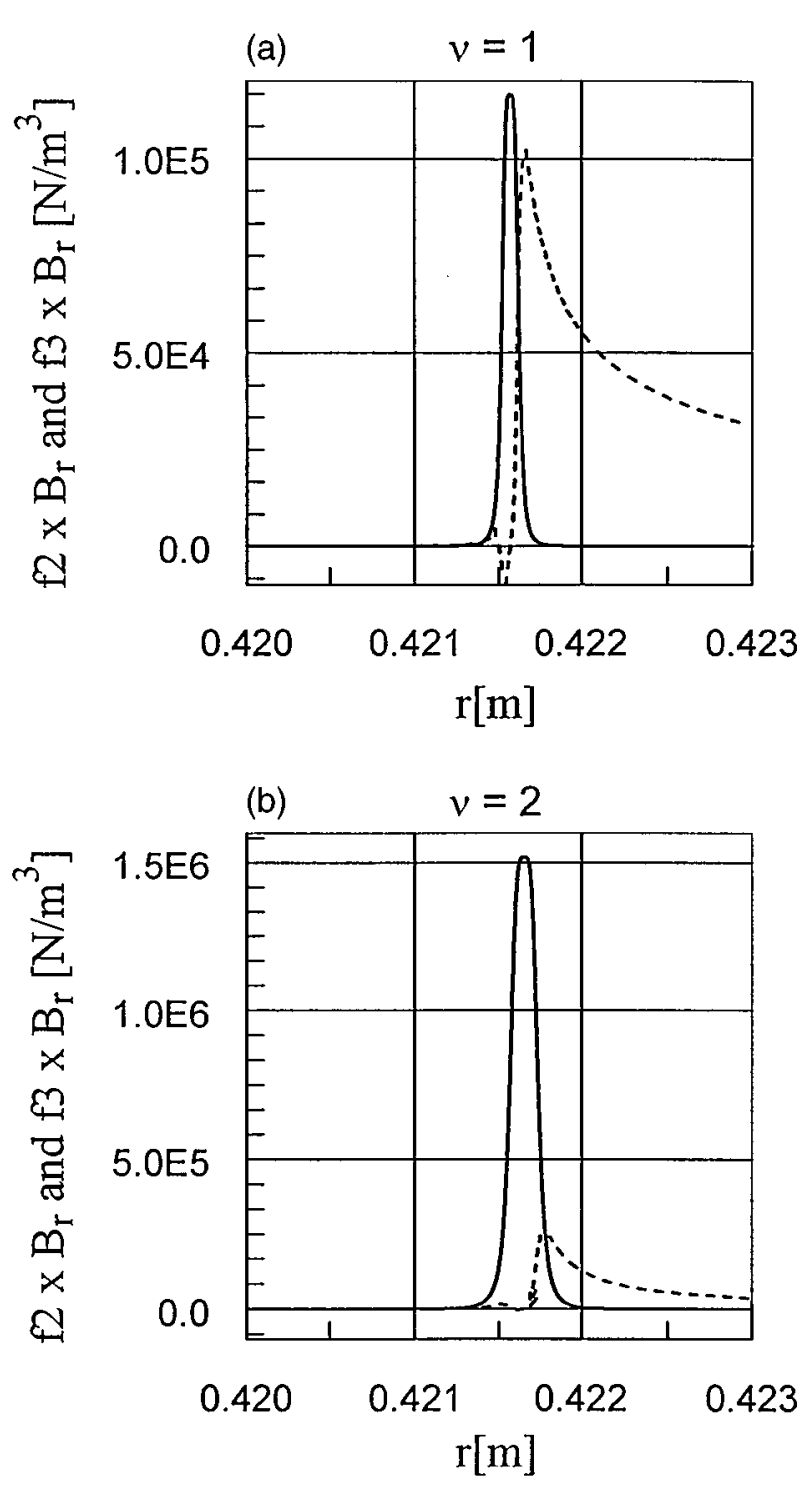

Figure 5. Contribution of the resonant term of Eq. 2 $\left(f 2 \times B_{r}\right)$ and of the term describing the interaction with the equilibrium current $\left(f 3 \times B_{r}\right)$ for the two values $\nu=1$ and 2. The solid line shows the term $f 2 \times B_{r}$ and the dotted one $f 3 \times B_{r}$.

A characteristic time of exchange between plasma edge and wall is given by the particle confinement time, a quantity which is experimentally available on TEXTOR. To simplify the treatment, a constant value of $\tau_{p}=30 \mathrm{~ms}$ is used:

$\nu^{*}=\frac{1}{\tau_{p}}=(1 / 0.03) \mathrm{s}^{-1}=33 \mathrm{~s}^{-1}$.

The mass $M$ is the total mass of the plasma because we assume that in the toroidal direction the whole plasma will rotate as a rigid body if no additional toroidal forces are applied (e.g. due to NBI). As particle content for the total plasma $N=2.4 \times 10^{20}$ particles is taken, which corresponds to a medium density value of TEXTOR-94 $\left(\bar{n}_{e}=4 \times 10^{19} \mathrm{~m}^{-3}\right.$, $n_{\text {-edge }}=1 \times 10^{19} \mathrm{~m}^{-3}$ ).

The force component in the toroidal direction, $F_{t}$, has been treated in the preceding section; the calculations were done, however, for a non-rotating plasma. The rotation is taken into account by writing $\left(F_{0}\right.$ being the force in a frame without plasma rotation):

$F_{t}=F_{0}\left(1-\frac{v}{v_{0}}\right)$

$v_{0}=\frac{R \Omega}{n_{\text {eff }}}=1.1 \times 10^{5} \mathrm{~m} / \mathrm{s}$

where $R=1.75 \mathrm{~m}$ is the major radius, $\Omega=2 \pi \times$ $10^{4} \mathrm{~s}^{-1}$ is the angular frequency of the DED field and $n_{\text {eff }}=1$ is the toroidal mode number under consideration. Here we have chosen the $m / n=3 / 1$ mode for the DED to obtain the highest plasma rotation. With these assumptions, the toroidal rotation velocity of the plasma is determined as:

$\frac{v_{\text {tor }}}{v_{0}}=\frac{\left(\tau_{p} / M\right)\left(F_{0} / v_{0}\right)}{1+\left(\tau_{p} / M\right)\left(F_{0} / v_{0}\right)}=0.66$.

This estimate shows that the DED field will most likely induce a substantial toroidal plasma rotation velocity; the plasma velocity, however, does not reach the full velocity of the perturbation field but remains at about half of this value.

\section{Considerations on radial electric field, current and poloidal velocity resulting from the poloidal force, with an estimate of the current}

The following considerations on the radial electric field and poloidal force are based on the revisited neoclassical transport theory [18-20]. In the revisited version, the gyroviscosity and the action of the energy transfer between electrons and ions on the temperature and density gradients along the field lines are taken into account. These additional terms then allow for radial electric currents and poloidal rotation of the plasma.

In the following estimations we take a constant poloidal force of $F_{p o l}=100 \mathrm{~N}$ to act on the whole plasma, corresponding to a force per particle of $f=F_{\text {pol }} / 2 \pi^{2} R a^{2} N$. If the average density is 


\section{K.H. Finken et al.}

$N=4 \times 10^{19} \mathrm{~m}^{-3}$, we obtain $f=3 \times 10^{-19} \mathrm{~N}$. In the following we use the unit vectors:

$\hat{\boldsymbol{n}}$ : unit vector parallel to $\boldsymbol{B}$,

$\hat{\boldsymbol{p}}$ : unit vector orthogonal to the magnetic flux surfaces (i.e. in the radial direction),

$\hat{\boldsymbol{b}}$ : unit vector in the direction of the binormal (i.e. $\perp \boldsymbol{B}$ but tangent to the flux surface).

The leading terms of the momentum equations for electrons and ions can be written as:

$$
\begin{aligned}
0= & -\hat{\boldsymbol{b}} \cdot \boldsymbol{\nabla} P_{e}-e N_{e}\left(\hat{\boldsymbol{b}} \cdot \boldsymbol{E}-B \hat{\boldsymbol{p}} \cdot \boldsymbol{U}_{e}\right)+N_{e} \hat{\boldsymbol{b}} \cdot \boldsymbol{f}_{e} \\
& +\hat{\boldsymbol{b}} \cdot \boldsymbol{R}
\end{aligned}
$$

(for electrons) where $\boldsymbol{f}_{e}$ is the force per particle and $\boldsymbol{R}$ is the friction force between electrons and ions, and

$$
\begin{aligned}
& m_{i} N_{i} \hat{\boldsymbol{b}} \cdot\left(\boldsymbol{U}_{i} \boldsymbol{\nabla} \boldsymbol{U}_{i}\right)=-\hat{\boldsymbol{b}} \cdot \nabla P_{i}-\hat{\boldsymbol{b}} \cdot\left(\boldsymbol{\nabla} \cdot \boldsymbol{\Pi}_{i}\right) \\
& \quad+e N_{i}\left(\hat{\boldsymbol{b}} \cdot \boldsymbol{E}-B \hat{\boldsymbol{p}} \cdot \boldsymbol{U}_{i}\right)+N_{i} \hat{\boldsymbol{b}} \cdot \boldsymbol{f}_{i}-\hat{\boldsymbol{b}} \cdot \boldsymbol{R}
\end{aligned}
$$

(for ions) where $\boldsymbol{\Pi}$ is the stress tensor. Adding these equations yields

$$
\begin{gathered}
m_{i} N_{i} \hat{\boldsymbol{b}} \cdot\left(\boldsymbol{U}_{i} \cdot \boldsymbol{\nabla} \boldsymbol{U}_{i}\right)=-\hat{\boldsymbol{b}} \cdot \boldsymbol{\nabla}\left(P_{i}+P_{e}\right)-\hat{\boldsymbol{b}} \cdot\left[\boldsymbol{\nabla} \cdot\left(\boldsymbol{\Pi}_{i}\right)\right] \\
+e N_{i} B\left(\hat{\boldsymbol{p}} \cdot \boldsymbol{U}_{e}-\hat{\boldsymbol{p}} \cdot \boldsymbol{U}_{i}\right)+N_{e}\left(\hat{\boldsymbol{b}} \cdot \boldsymbol{f}_{e}+\hat{\boldsymbol{b}} \cdot \boldsymbol{f}_{i}\right)
\end{gathered}
$$

The term $\hat{\boldsymbol{b}} \cdot \boldsymbol{\nabla}\left(P_{e}+P_{i}\right)$ is eliminated by performing a flux surface averaging. The flux surface average of the inertia and the stress tensor terms must balance the flux surface average of the external force term since the flux surface average of the radial current must vanish.

The force has the same sign for electrons and ions and amounts, as already stated, to $f=3 \times$ $10^{-19} \mathrm{~N} /$ particle. This would lead, in terms of order of magnitude, to a relative velocity between electrons and ions of

$\hat{\boldsymbol{p}} \cdot \boldsymbol{U}_{e}-\hat{\boldsymbol{p}} \cdot \boldsymbol{U}_{i}=-\left(\hat{\boldsymbol{b}} \cdot \boldsymbol{f}_{e}+\hat{\boldsymbol{b}} \cdot \boldsymbol{f}_{i}\right) / e B=1 \mathrm{~m} / \mathrm{s}$

if the plasma did not react. This drift velocity of ions and electrons in opposite radial directions corresponds to a total current of about $I_{r}=50 \mathrm{~A}$ if an edge electron density of $n_{e-e d g e}=1 \times 10^{19} \mathrm{~m}^{-3}$ is assumed (this is about the same current as is observed during polarization experiments).

\section{Radial electric field}

The ambipolar radial electric field can be obtained from Eq. (58) of Ref. [18]. Here the radial electric field is $E_{\psi} \equiv-h_{\psi}^{-1} \partial V^{(0)} / \partial \psi$; note that $E_{\psi}$ also appears via $U_{\|}^{(1)}$ to which it is related by the radial momentum equation $E_{r}=U_{\varphi} B_{\theta}-U_{\theta} B_{\varphi}+$ $\left(e N_{i}\right)^{-1} \partial P_{i} / \partial r$ and $U_{\varphi} \cong U_{\|}$. For an order of magnitude estimate, only the last two terms on the right hand side of the equation including $K^{*}$, which can be identified with

$K^{*}=-\Omega_{i}\left(B_{\theta} / B_{\varphi}\right) N\left(\hat{\boldsymbol{p}} \cdot \boldsymbol{U}_{e}-\hat{\boldsymbol{p}} \cdot \boldsymbol{U}_{i}\right)$

are balanced here. Hence

$$
\begin{aligned}
\frac{E_{r}}{B_{\varphi}} & =-0.625 \frac{B_{\theta}^{2}}{B_{\varphi}^{2}} \frac{L_{\perp}^{2}}{q^{2} a_{i}^{2}} \Omega_{i} \tau_{i}\left(\hat{\boldsymbol{p}} \cdot \boldsymbol{U}_{e}-\hat{\boldsymbol{p}} \cdot \boldsymbol{U}_{i}\right) \\
& =0.625 \frac{B_{\theta}^{2}}{B_{\varphi}^{2}} \frac{L_{\perp}^{2}}{q^{2} a_{i}^{2}} \Omega_{i} \tau_{i} \frac{\hat{\boldsymbol{b}} \cdot\left(\boldsymbol{f}_{e}+\boldsymbol{f}_{i}\right)}{e B}
\end{aligned}
$$

where $L_{\perp}$ is a characteristic gradient length scale and $a_{i}^{2}=T_{i} / m_{i} \Omega_{i}^{2}$; note that

$E_{r} / L_{\perp}^{2} \propto \hat{\boldsymbol{b}} \cdot \boldsymbol{f} / a_{i}^{2}$

In order to obtain a quantitative estimate we introduce for the ion collision time

$$
\frac{\tau_{i}}{[\mathrm{~s}]}=1.41 \times 10^{12} A_{i}^{1 / 2} T_{i}^{3 / 2}(\mathrm{eV})\left[Z_{e f f, i} N_{i}\left(\mathrm{~m}^{-3}\right)\right]^{-1} .
$$

Hence the following scaling:

$\frac{E_{r}}{B_{\varphi}} \propto \frac{B^{2} T_{i}^{1 / 2} \hat{\boldsymbol{b}} \cdot \boldsymbol{f}}{q^{4} A_{i}^{3 / 2} Z_{e f f, i} N_{i}} \frac{r^{2}}{R^{2}} L_{\perp}^{2}$

Assuming $q=3, r / R=1 / 4, B_{\theta} / B_{\varphi}=1 / 12$, $Z_{\text {eff }, i} n_{i}=2 \times 10^{19} \mathrm{~m}^{-3}, T_{i}=200 \mathrm{eV}, B=2.25 \mathrm{~T}$ and $f_{e, i}=3 \times 10^{-19} \mathrm{~N} /$ particle, one obtains

$\frac{\left(\frac{-E_{r}}{B_{\varphi}}\right)}{[\mathrm{m} / \mathrm{s}]} \approx 1.4 \times 10^{3}\left[\frac{L_{\perp}}{\mathrm{cm}}\right]^{2}$

and

$\frac{E_{r}}{[\mathrm{kV} / \mathrm{m}]} \approx 3\left[\frac{L_{\perp}}{\mathrm{cm}}\right]^{2}$

If the width of the ergodic zone is introduced for $L_{\perp}$, we obtain $E_{r} \approx(3-30) \mathrm{kV} / \mathrm{m}$, comparable to values reported in $\mathrm{H}$ mode [21] transport barriers and polarization [22] experiments.

\section{Conclusions}

Considerable progress has been made in recent years in the understanding of the static ergodic divertor [12], both theoretically and experimentally. It has been understood that the ergodic zone with very long 
magnetic field lines — which show (sub-)diffusive properties in a finite volume - and the laminar zone with open field lines are important, and that both regions have different and partially complementary properties. For analysing the ergodic zone and the transport in that region, powerful mapping methods have been developed.

The understanding of a rotating perturbation magnetic field, on the other hand, is very new ground and this contribution is one of the first steps into the field. A first basic question concerns the physics of the reconnection of the magnetic field in an ergodized layer and what assumption to make about the thickness of the induced shielding current layer there. An earlier model of the shielding current is extended and new results are presented for the torque exerted by the external field on the plasma.

These force components are used as input into the revisited neoclassical theory and tentative first steps are taken towards finding out whether the forces transferred from the DED are sufficient to impose a substantial plasma rotation. Even though not all terms of the basic equations were evaluated, it seems very likely that these forces are indeed large enough to obtain a toroidal rotation of the whole plasma column with velocities of the order of the DED rotation velocity. The radial electric field is of the same order of magnitude as that we are used to in polarization $\mathrm{H}$ mode.

\section{References}

[1] Special Issue: Dynamic Ergodic Divertor, Fusion Eng. Des. 37 (1997) 335-450.

[2] Kobayashi, M., et al., Nucl. Fusion 40 (2000) 181.

[3] Foster, M.S., McCool, S.C., Wooton, A.J., Nucl. Fusion 35 (1995) 329.

[4] Lichtenberg, J.A., Lieberman, M.A., "Regular and stochastic motion", Applied Mathematical Sciences, Springer, New York (1983) 38.
[5] Evans, T.E., et al., J. Nucl. Mater. 145-147 (1987) 812.

[6] Grosman, A., et al., J. Nucl. Mater. 196-198 (1992) 59.

[7] Takamura, S., et al., Phys. Fluids 30 (1987) 144.

[8] Eich, T., Reiser, D., Finken, K.H., Nucl. Fusion 40 (2000) 1757.

[9] Abdullaev, S.S., Finken, K.H., Kaleck, A., Spatschek, K.H., Phys. Plasmas 5 (1998) 196.

[10] Abdullaev, S.S., Finken, K.H., Spatschek, K.H., Phys. Plasmas 6 (1999) 153.

[11] Finken, K.H., Abdullaev, S.S., Kaleck, A., Wolf, G.H., Nucl. Fusion 39 (1999) 637.

[12] Ghendrih, P., Grosman, A., Capes, H., Plasma Phys. Control. Fusion 38 (1996) 1653.

[13] Finken, K.H., Eich, T., Kaleck, A., Nucl. Fusion 38 (1998) 515

[14] Finken, K.H., Nucl. Fusion 39 (1999) 707.

[15] Faulconer, D.W., Koch, R., Fusion Eng. Des. 37 (1997) 399.

[16] Wesson, J., Tokamaks, Clarendon Press, Oxford (1997) Ch. 3.7.

[17] Hahm, T.S., Burrell, K.H., Phys. Plasmas 2 (1995) 1648 .

[18] Rogister, A., Phys. Plasmas 1 (1994) 619.

[19] Claasen, H.A., Gerhauser, H., Rogister, A., Yarin, C., Phys. Plasmas 7 (2000) 3699.

[20] Engelmann, F., Nocentini, A., Nucl. Fusion 16 (1976) 694.

[21] Burrell, K.H., et al., Plasma Phys. Control. Fusion 34 (1992) 1859.

[22] Weynants, R.R., Jachmich, S., Van Oost, G., Plasma Phys. Control. Fusion 40 (1998) 635.

(Manuscript received 4 October 2000

Final manuscript accepted 22 January 2001)

E-mail address of K.H. Finken:

k.h.finken@fz-juelich.de

Subject classification: B0, Tt; I0, Tt 